\title{
Selected Abstracts of the TEEPGI 2014 Workshop
}

8th Annual Perioperative and Critical Care Transesophageal Echocardiography workshop 28th Feb. to 2nd March 2014, Advanced Cardiac Centre, PGIMER, Chandigarh, India

\section{latrogenic Mitral Valve Perforation Following CABG and Aortic Valve Replacement: A Rare Complication Detected by Post-Bypass Transesophageal Echocardiography}

\section{${ }^{1}$ Ravi Raj, ${ }^{2}$ Goverdhan Dutt Puri}

${ }^{1}$ Senior Resident, Department of Anesthesia and Intensive Care, Postgraduate Institute of Medical Education and Research Chandigarh, India

2Professor, Department of Anesthesia and Intensive Care, Postgraduate Institute of Medical Education and Research, Chandigarh India

\begin{abstract}
Mitral valve perforation is most commonly due to infective endocarditis. latrogenic mitral valve perforation following aortic valve replacement has not been described previously. A 57 years male patient presented with complaints of progressive dyspnea on exertion and occasional palpitations. A preoperative diagnosis of severe aortic stenosis, sclerodegenerative aortic valve with normal left ventricle function was made on transthoracic echocardiography. A coronary angiogram showed single vessel disease involving proximal left anterior descending artery causing $80 \%$ stenosis. The patient was planned for aortic valve replacement (AVR) and CABG. Pre bypass TEE showed bicuspid aortic valve, thick, calcified, severe aortic stenosis and normal left ventricle systolic function. Mitral valve was morphologically normal with mild central mitral regurgitation jet. Patient underwent CABG and AVR under cardiopulmonary bypass support.
\end{abstract}

Post CPB TEE examination showed 2 jets of mitral regurgitation in midesophageal aortic long-axis view (Fig. 1). There was a mild central MR jet and an additional mild MR jet from the body of anterior mitral leaflet. Transgastric short axis view showed turbulence in the region of A1 scallop of anterior mitral leaflet. We present the intraoperative TEE images of the patient with a discussion on the role of TEE in detection of mitral valve perforation and surgical decision making.

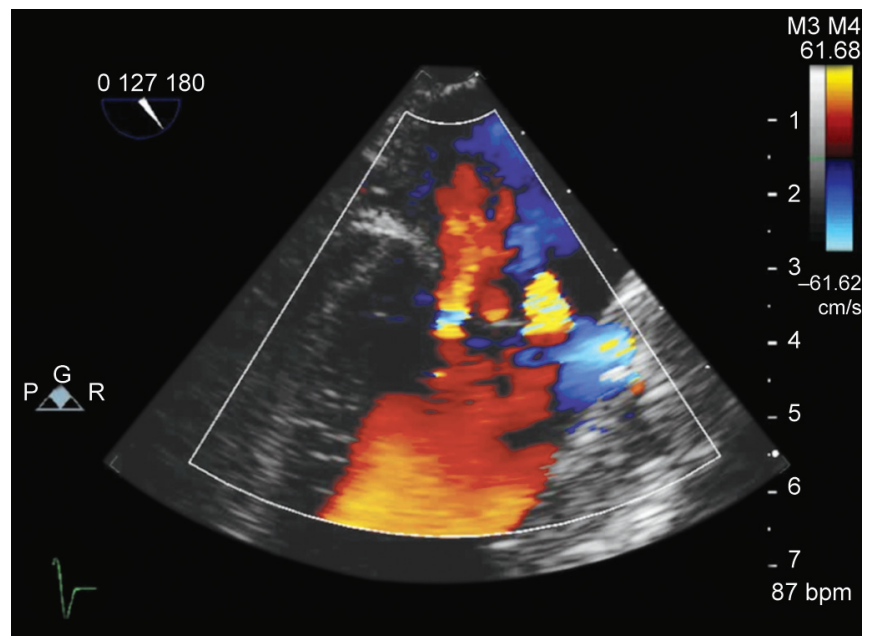

Fig. 1: Midesophageal aortic long-axis view showing 2 jets of MR in post-bypass period (one central jet at co-optation point and an additional jet from body of anterior mitral leaflet) 


\title{
Foreign Body in the Ascending Aorta
}

\author{
${ }^{1}$ Rupa Sreedhar, ${ }^{2}$ Roshit Chandran, ${ }^{3}$ Reshmi Liza Jose, ${ }^{4}$ VG Shrinivas, ${ }^{5}$ Jay Kumar, ${ }^{6}$ Simran \\ ${ }^{1,4}$ Professor, Department of Anesthesiology, Sree Chitra Tirunal Institute for Medical Sciences and Technology, Thiruvananthapuram \\ Kerala, India \\ ${ }^{2}$ Senior Resident, Department of Cardiovascular and Thoracic Surgery, Sree Chitra Tirunal Institute for Medical Sciences and \\ Technology, Thiruvananthapuram, Kerala, India \\ ${ }^{3}$ Senior Resident, Department of Anesthesiology, Sree Chitra Tirunal Institute for Medical Sciences and Technology \\ Thiruvananthapuram, Kerala, India \\ ${ }^{5}$ Professor and Head, Department of Cardiovascular and Thoracic Surgery, Sree Chitra Tirunal Institute for Medical Sciences \\ and Technology, Thiruvananthapuram, Kerala, India \\ ${ }^{6}$ Senior Resident, Department of Anesthesia, Sree Chitra Tirunal Institute for Medical Sciences and Technology, Thiruvananthapuram \\ Kerala, India
}

\section{ABSTRACT}

Aim: We intend to report a case of foreign body in the ascending aorta without occurrence of cardiac tamponade.

Case report: A 22-year-old male who was involved in a road traffic accident 7 months prior to the visit to our Institute, sustained a fracture of the right clavicle, for which he underwent K-wiring. After a period of 2 months the pins were scheduled for removal. However at manipulation, one of the pins got further embedded in the bone and he was rescheduled for removal of the pin under anesthesia.

He soon developed dyspnea on exertion functional class II, with pedal edema. Chest X-ray revealed the presence of pleural effusion, and that the pin had migrated into his chest. Lateral X-ray was more informative so as to reveal the location within the cardiac silhouette.

Echo showed a hyperechoic shadow in the ascending Aorta which was tenting the right coronary cusp (RCC) and causing severe aortic regurgitation (AR). Flouroscopy revealed the pin to be moving with each heart beat. The patient was taken up for exploration.

The patient surprisingly had no pericardial effusion. Externally the point of entry was at the right sterno-clavicular joint. Internally, the nail had penetrated the pericardium above the superior vena cava (SVC) and the ascending aorta on its anterior surface forming a saccular aneurysm. Transesophageal echocardiography (TEE) showed the nail to be tenting the RCC and causing severe AR. The nail was removed. TEE showed persistent AR and damaged RCC. It was therefore decided to go on cardiopulmonary bypass and examine the aortic valve. On examination, the RCC was found to be torn and not feasible for repair. Therefore, the aortic valve was replaced with $19 \mathrm{~mm}$ Chitra TTK ${ }^{\circledR}$ heart valve prosthesis.

In our patient, the foreign body could be easily taken out by taking a purse string around the site of entry into the aorta to control it; we needed to go on bypass only to replace the aortic valve.

Results: Three months after surgery, the patient is doing well and is in functional class I.

Conclusion: In management of patients with foreign body in the aorta, it is imperative to localize the foreign body using TEE prior to embarking on surgical expedition of the vascular system because foreign bodies which get lodged in the vascular system may embolized. The TEE images guided the surgery throughout its course. They perfectly delineated the site of impaction and the mechanism of AR.

\section{Stuck Leaflet of ST Jude Mitral Prosthesis after Cardiopulmonary Bypass: Detection of Mechanism using Intraoperative 3D Echocardiography}

\author{
${ }^{1}$ Shrinivas Gadhinglajkar, ${ }^{2}$ Suddhadeb Roy, ${ }^{3}$ Rupa Sreedhar \\ ${ }^{1,3}$ Professor, Department of Anesthesia, Sree Chitra Tirunal Institute for Medical Sciences and Technology, Thiruvananthapuram \\ Kerala, India \\ ${ }^{2}$ Senior Resident, Department of Cardiothoracic and Vascular Anesthesia, Sree Chitra Tirunal Institute for Medical Sciences and \\ Technology, Thiruvananthapuram, Kerala, India
}

\begin{abstract}
Background: Mechanical prosthesis dysfunction at mitral position after cardiopulmonary bypass (CPB) may occur due to manufacturing defect in the prosthesis or entrapment of sutures, residual portion of native leaflet or any other loose tag of tissue intervening between the leaflet and the annular metallic stent. Diagnosing the exact mechanism of prosthesis dysfunction on transesophageal echocardiography (TEE) enables the surgeon to take decision regarding reinstitution of CPB and planning the succeeding surgical steps. Intraoperative 3D echocardiography can diagnose the problem because of its capacity to virtually crop the data, rotate it and present the images to the surgeons in anatomical fashion. We present our experience of intraoperative 3D-TEE that detected the cause of leaflet malfunction.
\end{abstract}


Materials and methods: A 45-year-old man was subjected to MV replacement using St Jude bileaflet prosthesis for rheumatic mitral stenosis. Most of the diseased valve was excised along with chordae, although some portion of posterior leaflet near left side of the annulus was retained as it was calcific and firmly adherent to the annulus. After weaning the patient from CPB, the left-sided leaflet was found immobile. The 2D-TEE showed immobility of one of the leaflets, although could not detect the mechanism of leaflet immobility. Virtual cropping through left atrium and left ventricle using intraoperative 3D-TEE revealed a tag of tissue covering the prosthesis at 8 o'clock to 11 o'clock position, causing leaflet immobility. This finding was confirmed by virtual prosthetectomy performed on anterior aspect and left side of the annulus. The CPB was re-established to rectify the problem.

Results: Surgeon immediately detected the tag of tissue on reinstituting the bypass and removed it, which resulted in satisfactory movement of both leaflets in the post-CPB period.

Conclusion: Exact mechanism of prosthesis leaflet dysfunction could be detected on intraoperative 3D-TEE, but not on 2D-TEE. It guided the surgeon to deal with the problem without delay and avoiding undue surgical exercise.

\title{
Surgical Repair of Partial Atrioventricular Septal Defect: Role of 2D vs 3D Echocardiography
}

\author{
${ }^{1}$ Suddhadeb Roy, ${ }^{2}$ Shrinivas Gadhinglajkar, ${ }^{3}$ Rupa Sreedhar \\ ${ }^{1}$ Senior Resident, Department of Cardiothoracic and Vascular Anesthesia, Sree Chitra Tirunal Institute for Medical Sciences and \\ Technology, Thiruvananthapuram, Kerala, India \\ ${ }^{2,3}$ Professor, Department of Anesthesia, Sree Chitra Tirunal Institute for Medical Sciences and Technology, Thiruvananthapuram \\ Kerala, India
}

\begin{abstract}
Background: Role of intraoperative 3D-TEE is well established in adult patients undergoing mitral valve repair. However, there is a paucity of literature on its utility during valve repair in patients with atrioventricular septal defects (AVSD). Unlike a normal mitral valve, which is composed of anterior mitral leaflet (AML) and posterior mitral leaflet (PML), the left $A V$ valve in a patient with partial AVSD has morphologically different leaflets namely left superior leaflet (LSL), left inferior leaflet (LIL) and left lateral leaflet (LLL). The cleft AML is the gap between LSL and LIL, which is closed during surgery. It is difficult to assess the left AV valve repair on intraoperative 2D-TEE. We present our experience with intraoperative 3D-TEE during the surgical repair of AVSD in adult.
\end{abstract}

Materials and methods: A 26-year-old lady was operated for correction of partial AVSD with cleft AML. Preoperative transthoracic echocardiography revealed ostium primum ASD $(20 \mathrm{~mm} \times 30 \mathrm{~mm})$, cleft between LSL and LIL, moderate mitral regurgitation (MR) and a normal tricuspid valve. After anesthesia induction, heart was inspected using intraoperative 3D-TEE. The midesophageal window showed a large left atrium, a common AV valve, ostium primum ASD and moderate MR. Although, the cleft in AML could be identified on transgastric views, leaflet orientation was ambiguous. Intraoperative 3D-TEE could demonstrate the morphology of all leaflets and revealed the cleft between LSL, LIL with orientation of LSL close to aortic valve. The cleft was approximated with 6-0 prolene. Commissuroplasty was performed for both mitral commissures and the ASD closed with tanned pericardial patch.

Results: The closure of cleft could be clearly verified on intraoperative 3D. The mitral valve was mildly regurgitant without any iatrogenic stenosis.

Conclusion: The intraoperative 3D-TEE has an upper edge over the 2D-TEE in identifying individual leaflets in AVSD, verifying closure of cleft and grade of MR.

\section{Postoperative Aortic and Mitral Valve Replacement with Ruptured Para-aortic Abscess: Transesophageal Echocardiography Guided Surgical Reintervention}

\footnotetext{
${ }^{1} \mathrm{R}$ Uvaraj, ${ }^{2}$ Shrinivas Gadhinglajkar, ${ }^{3}$ Rupa Sreedhar

${ }^{1}$ Senior Resident, Department of Cardiothoracic and Vascular Anesthesia, Sree Chitra Tirunal Institute for Medical Sciences and Technology, Thiruvananthapuram, Kerala, India

${ }^{2,3}$ Professor, Department of Anesthesia, Sree Chitra Tirunal Institute for Medical Sciences and Technology, Thiruvananthapuram Kerala, India
}

\section{ABSTRACT}

Background: Infective endocarditis after mitral and aortic valve replacement may be associated with aortic root abscess, which may rupture into left ventricular outflow tract (LVOT) or left atrium (LA). Surgical repair in the presence of infective endocarditis 
complicated by aortic root abscess may be difficult due to distorted anatomy and friable valve tissue. We are reporting our experience in a patient, who was operated for double valve replacement (DVR). The course of surgery was guided by transesophageal echocardiography (TEE).

Materials and methods: A 45-year-old patient, who was operated for DVR, presented 4 months after surgery with features of infective endocarditis. The transthoracic echocardiography revealed severe obstruction of mitral valve prosthesis (pressure gradient of $45 / 18 \mathrm{~mm} \mathrm{Hg}$ ), paravalvular leak and pulmonary hypertension. Both aortic and mitral valve prostheses were functioning well. Intraoperative TEE showed that the aortic root abscess had ruptured into LVOT and LA. It formed fistula between LVOT and LA via cavity of root abscess. Both aortic and mitral annuli were unstable as the aortomitral fibrous continuity had broken. After establishing cardiopulmonary bypass and replacing mitral valve, the surgeon was in dilemma, whether the aortic valve need replacement. As aortic and mitral valve annuli were found unstable on TEE, the aortic valve was explanted, the annuli were restrengthened and new aortic valve was replaced.

Observations: Patient had successful surgical repair with adequate prosthetic function. He was discharged 10 days after the surgery.

Conclusion: The TEE can diagnose infective endocarditis related complications, decide about the course of surgery and also evaluate adequacy of surgical repair.

\section{Residual AR Following VSD Repair}

\section{${ }^{1}$ BPS Ghumman, ${ }^{2}$ Goverdhan Dutt Puri}

${ }^{1}$ Senior Resident, Department of Anesthesia and Intensive Care, Postgraduate Institute of Medical Education and Research Chandigarh, India

${ }^{2}$ Professor, Department of Anesthesia and Intensive Care, Postgraduate Institute of Medical Education and Research, Chandigarh India

\section{ABSTRACT}

Background: Three years old female acyanotic child with complaints of rapid breathing and episodes of frequent upper respiratory tract infections since birth with preoperative pulse of 110 per minute regular, BP $90 / 60 \mathrm{~mm} \mathrm{Hg}$, respiratory rate of $24 / \mathrm{min}$. X-ray chest showed cardiomegaly with CT ratio of 0.6 , with signs of pulmonary artery hypertension. ECG showed right axis deviation with incomplete right bundle branch block. Transthoracic echocardiography showed perimembranous VSD with outlet extension of $6 \mathrm{~mm}$ size with left to right shunt with peak systolic gradient across VSD of $80 \mathrm{~mm} \mathrm{Hg}$. LA and LV were dilated. There was mild TR with RVSP of $22 \mathrm{~mm} \mathrm{Hg}$. Patient had mild aortic regurgitation (AR) with AR jet height / LV outflow track diameter of 3/10 (Fig. 1).

Surgery planned was VSD closure and assessment of aortic valve in intraoperative period by TEE.

Baseline TEE performed in OT confirmed the diagnosis. The VSD patch closure was performed on CPB. Post CPB TEE showed no leaks across the VSD patch but AR had increased with eccentric jet with increased AR jet height / LVOT height ratio of $8 / 12 \mathrm{~mm}$, with pressure half time of $320 \mathrm{msec}$ with flow reversal in descending aorta. Hence AR had increased from mild to moderate grade after VSD closure. In view of increased AR decision to repair the aortic valve on second run of CPB was taken. On opening the aorta, right coronary cusp was found to be elongated and prolapsed. The defect was repaired using Trusler technique of repair.

Patient came off CPB uneventfully for the second time. AR was reduced to mild grade after repair with PHT of $794 \mathrm{msec}$ and AR jet height/ LVOT height ratio of 5/14 (Fig. 2).

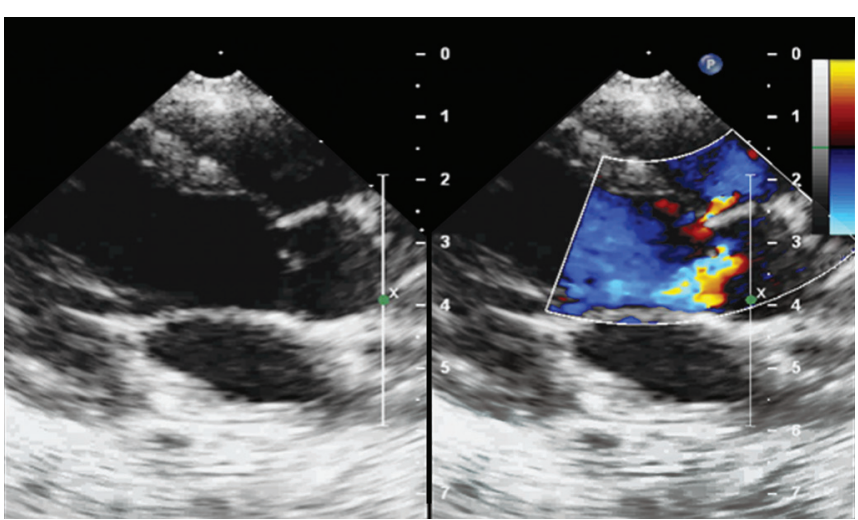

Fig. 1: Preoperative parasternal long-axis view on TTE showing VSD with AR

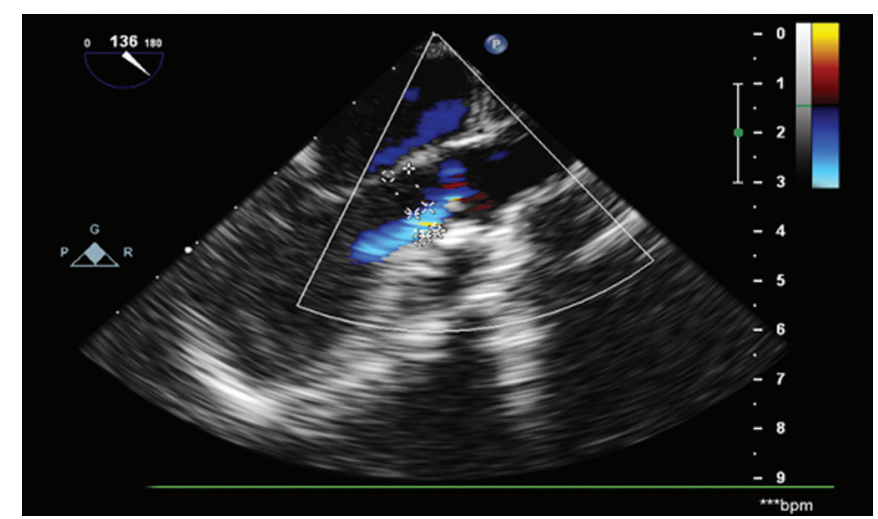

Fig. 2: Post 2nd CPB TEE ME long-axis view AR assessed post Trusler's repair 


\section{Perioperative Transthoracic and Transesophageal Echocardiography Assessment for Tetralogy of Fallot Repair: An Interim Analysis}

\section{${ }^{1}$ Ravi Raj, ${ }^{2}$ Aveek Jayant, ${ }^{3}$ Goverdhan Dutt Puri}

${ }^{1}$ Senior Resident, Department of Anesthesia and Intensive Care, Postgraduate Institute of Medical Education and Research Chandigarh, India

${ }^{2}$ Assistant Professor, Department of Anesthesia and Intensive Care, Postgraduate Institute of Medical Education and Research Chandigarh, India

${ }^{3}$ Professor, Department of Anesthesia and Intensive Care, Postgraduate Institute of Medical Education and Research, Chandigarh India

\section{ABSTRACT}

Tetralogy of fallot (TOF) is the most common cyanotic congenital heart disease. Perioperative transesophageal echocardiography (TEE) is an invaluable tool which can provide anatomic details required for planning surgical repair. However, pulmonary valve and branch pulmonary arteries, sometimes can be better seen on transthoracic echocardiography (TTE) due to their proximity to chest wall and interposition of left bronchus between esophagus and left pulmonary artery (LPA) during TEE examination. Evaluation of adequacy of repair and especially right ventricle function by post bypass TEE is invaluable for postoperative management.

Thirty patients with preoperative diagnosis of TOF or TOF physiology scheduled for corrective surgery were included for analysis. A detailed baseline TTE was performed in OR after induction of GA. A TEE probe appropriate for body weight was inserted for pre- and post-bypass evaluation. TTE was repeated in ICU for assessing adequacy of repair and biventricular function.

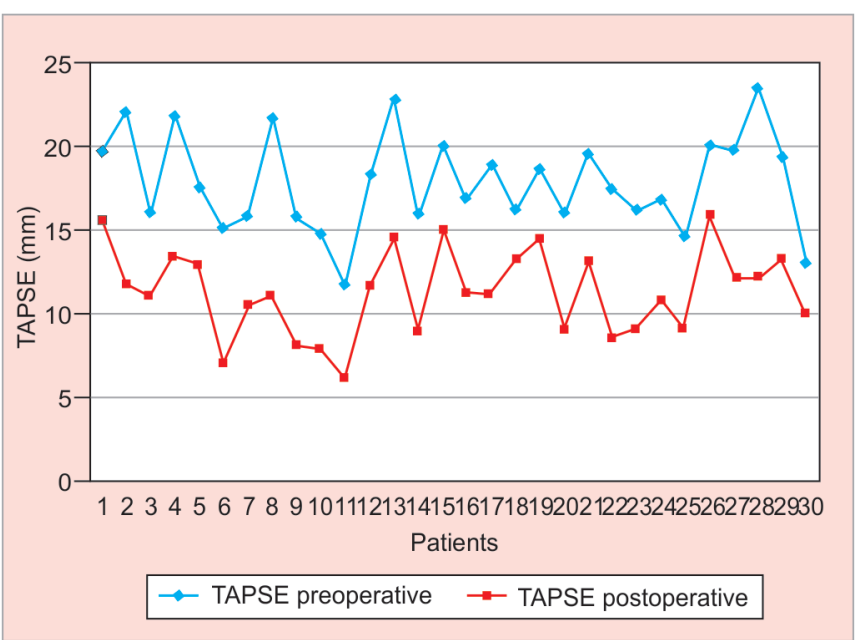

Fig. 1: Tricuspid annulus peak systolic excursion (TAPSE) values in pre- and postoperative period

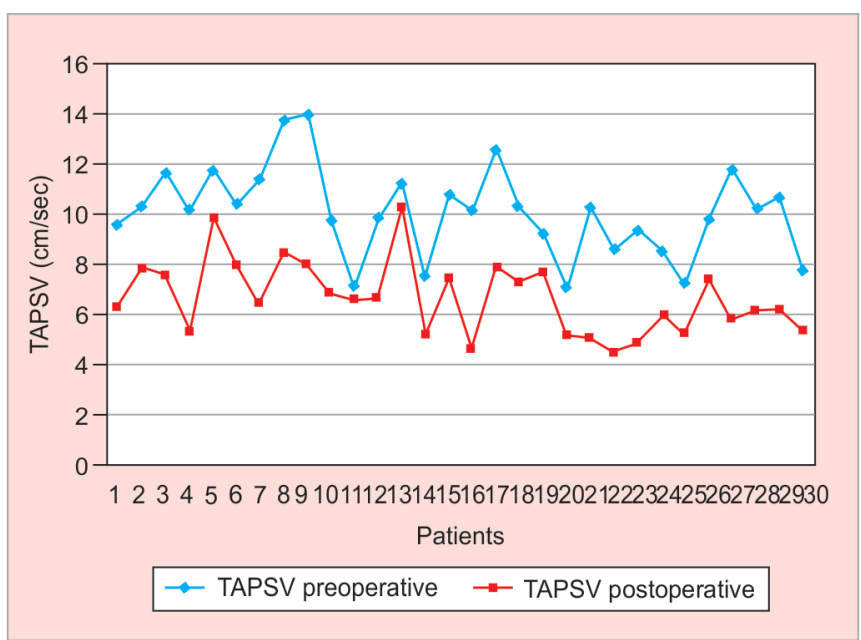

Fig. 2: Tissue Doppler imaging derived tricuspid annulus peak systolic velocity (TAPSV) in pre- and postoperative period

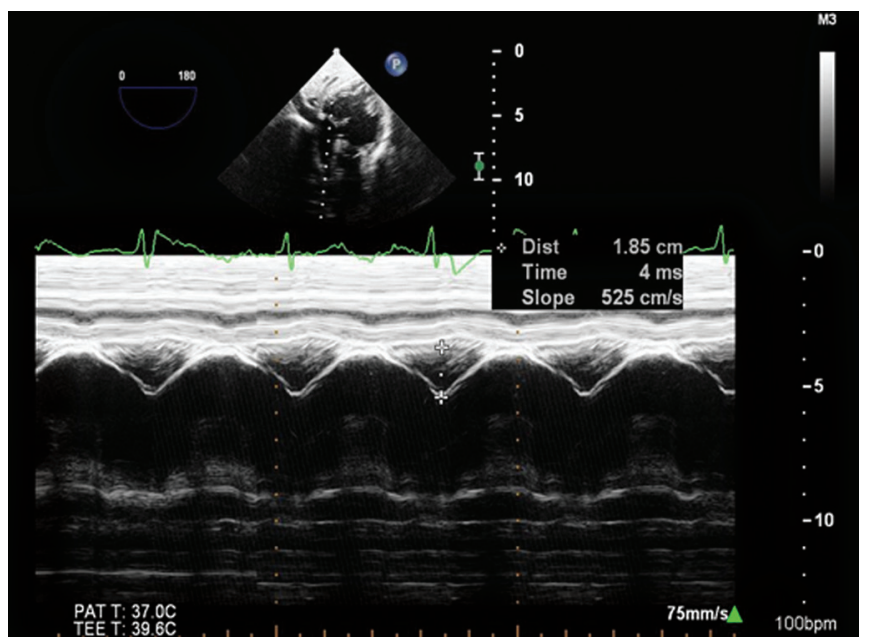

Fig. 3: Deep transgastric 4 chamber view showing estimation of tricuspid annulus peak systolic excursion (TAPSE) using M-mode echocardiography 
Both TTE and TEE were equally reliable for evaluation of right ventricle outflow tract, pulmonary valve and main pulmonary artery. Satisfactory evaluation of branch pulmonary arteries by TTE was possible in 28 (out of 30, 93.3\%) patients, while by TEE, especially, LPA was possible in only 14 (out of $30,46.67 \%$ ) patients. Right ventricle (RV) function parameters, i.e. tricuspid annulus peak systolic excursion (TAPSE) and tissue Doppler derived tricuspid annulus peak systolic velocity (TAPSV) decreased significantly after surgical repair (Figs 1 and 2). However, RV function parameters were not related to duration of mechanical ventilation or ICU stay.

\title{
Role of TEE in Detection of Residual Tumor in Perioperative Period in RCC with Cavoatrial Tumor Thrombus
}

\author{
${ }^{1}$ Sundar Negi, ${ }^{2}$ Ravi, ${ }^{3}$ A Misra, ${ }^{4}$ Goverdhan Dutt Puri \\ ${ }^{1}$ Senior Resident, Department of Anesthesia and Intensive Care, Postgraduate Institute of Medical Education and Research \\ Chandigarh, India \\ ${ }^{2,3}$ Assistant Professor, Department of Anesthesia and Intensive Care, Postgraduate Institute of Medical Education and Research \\ Chandigarh, India \\ ${ }^{4}$ Professor, Department of Anesthesia and Intensive Care, Postgraduate Institute of Medical Education and Research, Chandigarh, India
}

\section{ABSTRACT}

Surgical resection for renal cell carcinoma (RCC) extending into the inferior vena cava (IVC) and right atrium impose a challenge to the anesthesiologist and may require the use of veno-venous or cardiopulmonary bypass (CPB). The most serious and feared complication is embolization of the tumor thrombus during mobilization of the tumor causing a massive pulmonary embolism. Transesophageal echocardiography (TEE) can provide accurate identification and definition of the cranial extent of the tumor thrombus, but may also provide continuous monitoring of the hemodynamic status and any cardiac complications during surgical manipulation of tumor. Present report describes the use of TEE in recognizing not only the extent of the thrombus (Fig. 1) but also the adequacy of removal of the tumor thrombus after removal of tumor thrombus (Fig. 2) during right radical nephrectomy.

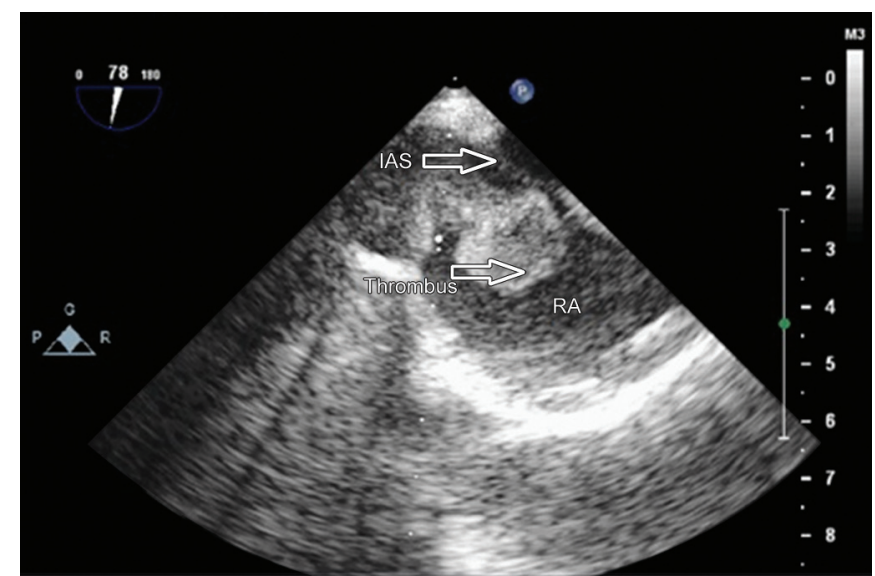

Fig. 1: Bicaval view showing tumor extending into right atrium

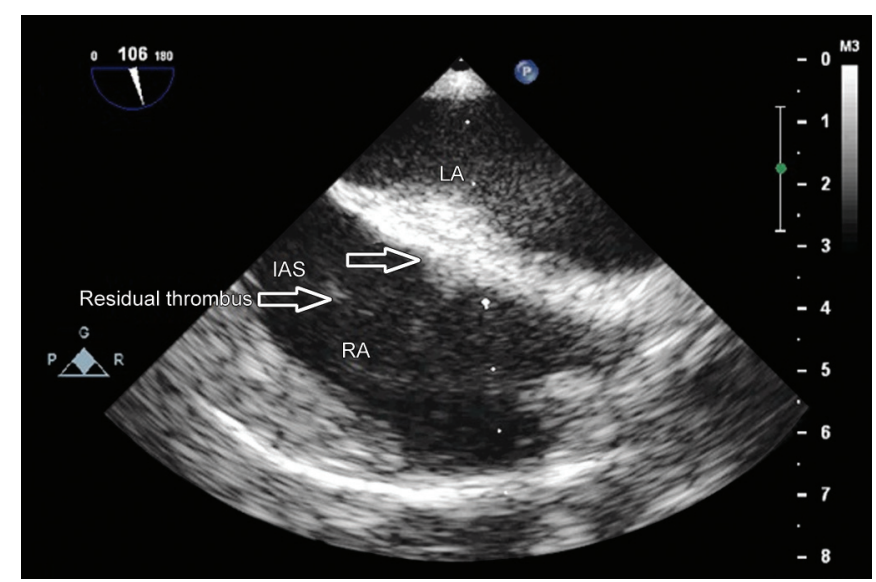

Fig. 2: Modified bicaval view showing residual tumor at RA IVC junction

\section{Role of TEE in Improving Diagnostic Accuracy of Congenital Heart Disease}

\footnotetext{
${ }^{1}$ Balbir Kumar, ${ }^{2}$ Madhavan J Sethu, ${ }^{3}$ Goverdhan Dutt Puri

${ }^{1}$ Junior Resident, Department of Anesthesia and Intensive Care, Postgraduate Institute of Medical Education and Research Chandigarh, India

${ }^{2}$ Senior Resident, Department of Anesthesia and Intensive Care, Postgraduate Institute of Medical Education and Research Chandigarh, India

${ }^{3}$ Professor, Department of Anesthesia and Intensive Care, Postgraduate Institute of Medical Education and Research, Chandigarh India
}

\section{ABSTRACT}

A 6-year-old child presented with dyspnea on exertion and failure to thrive. Physical examination was normal except for a continuous murmur at mitral area. Transthoracic echocardiography (TTE) revealed a large $6 \mathrm{~mm}$ PM VSD with left to right and 
single parachute papillary muscle. There was no coarctation of aorta. Intraoperative transesophageal echocardiography (TEE) revealed flow acceleration above the mitral valve with a supramitral ring which could not be delineated in TTE (Fig. 1). A gradient of $20 \mathrm{~mm} \mathrm{Hg}$ was noted in the peak radial and femoral artery pressures when cannulated intraoperatively. Anesthetic and cardiopulmonary bypass were as per standard institute protocol. During surgery the surgeon resected the supramitral ring and also noticed a subaortic membrane, which was resected. The same was found in the retrospective review of images, albeit very small. It also completed the necessary components for Shone complex ${ }^{1}$ - parachute mitral valve (TTE), supramitral ring (TEE) and subaortic membrane (retrospective diagnosis). TEE has been found to have an incremental value in the diagnosis of Shone complex. ${ }^{2}$ The use of TEE in congenital cases may increase the diagnostic yield in children and helps finalize the surgical plan.

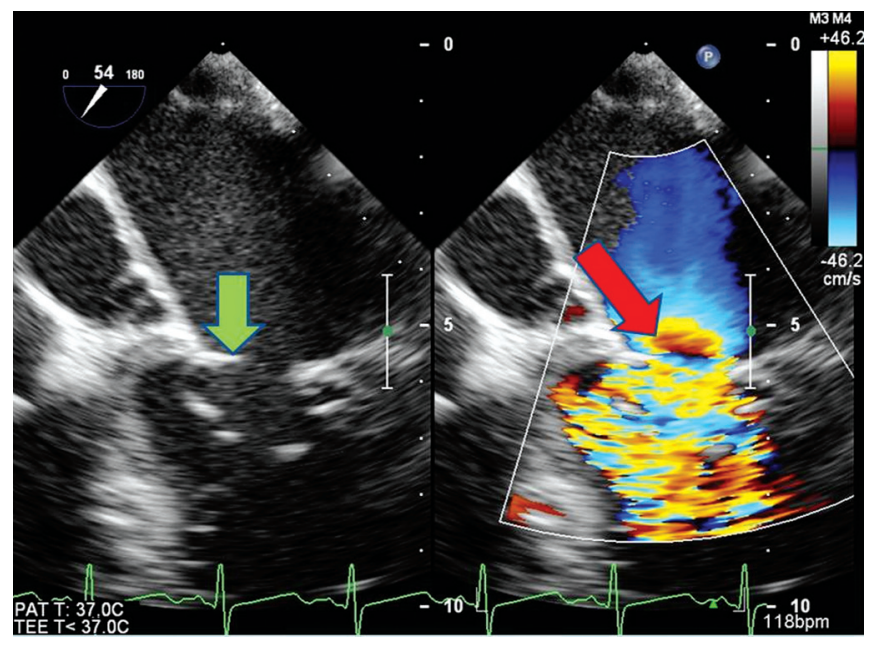

Fig. 1: Midesophageal commissural view showing supramitral ring (green arrow) and flow acceleration with proximal isovelocity surface area (PISA) formation on color Doppler (red arrow). The PISA is formed well above the level of mitral valve at the level os supramitral ring

\section{REFERENCES}

1. Shone JD. Sellers RD, Anderson RC, Adams P, Lillehei CW, Edwards JE. The developmental complex of 'parachute mitral valve,' supravalvular ring of left atrium, subaortic stenosis and coarctation of aorta.' Am J of Cardiolo 1963;11:714-725.

2. Moustafa SE, Lesperance J, Rouleau JL, Gosselin G. A forme frust of Shone's anomaly in a 65-year-old patient. McGill J Medi 2008;11(1):19-21. 